\title{
Design of high performance DC motor actuated cable driving system for compact devices
}

\author{
Chawaphol Direkwatana, Jackrit Suthakorn \\ Departement of Biomedical Engineering, Faculty of Engineering, Mahidol University, Thailand
}

\begin{tabular}{l} 
Article Info \\
\hline Article history: \\
Received Jan 6, 2020 \\
Revised Apr 3, 2020 \\
Accepted May 11, 2020 \\
\hline
\end{tabular}

\section{Keywords:}

Cable transmission

Fleet angle

Motion control

Precise transmission

Robotics

Wire-driven transmission

\begin{abstract}
The cable transmission is widely used in the remote operation or complex geometry with high stiffness and low backlash. Larger drum is required to reduce and error of transmission in long stroke. An error of the desired position occurs due to the fleet angle while cable winding on a drum. Therefore, a new cable driving module which overcomes this problem is proposed. A new cable driving module with a sliding platform is connected to the actuator unit. A motion of the sliding platform is corresponding to a screw rod connected to an actuator. The precision of the driving system is measured by a high-resolution rotatory encoder and high gear ratio actuator. Results are measured by load and error of the system. A load of system shows a performance of overall translation and rotation of the drum at different speeds. An error of the system is measured from forward and reverse direction by increasing and decreasing the number of turns with constant speed. A system has an average load consumption along a long stroke of cable winding which has no significant problem on the screw platform. Multiple turns have low error value in specific and continuous turn in forward and reverse motion. A new cable driving system is proved in precision movement. The fleet angle is eliminated in new mechanism. Along with a constraint motion, there is no significant change in load consumption. An error is low value in a different direction of movement. Hence, a new design of cable transmission can perform in high performance and small size of the system.
\end{abstract}

This is an open access article under the CC BY-SA license.

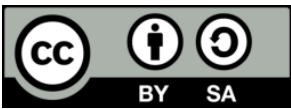

Corresponding Author:

Jackrit Suthakorn,

Center for Biomedical and Robotics Technology,

Departement of Biomedical Engineering, Faculty of Engineering, Mahidol University

25/25 Puttamonthon 4 Road, Salaya, Nakorn Pathom, Thailand 73170

Email: jackrit.sut@mahidol.ac.th jackrit@bartlab.org

\section{INTRODUCTION}

Power transmission system is a process to transmit motion from one shaft to another by connecting between them such as gear, wire rope and chain. Cable transmission is an alternative method to others transmission system in small and large system. It is used primarily in low speed applications. The advantages of cable transmission are high stiffness, high strength, high power to-weight ratio, low friction and no backlash. It requires low maintenance due to surface lubrication and can be used over long distance. According to flexibility, it can be used in normal and complex route of transmission.

Cable transmission has been widely used in many applications such as medical device, aerospace, robotics and in many research areas. In mechanism, cable transmission can be used in complex structure in such as spherical mechanism and parallelogram [1-3]. In robot arm, the size and weight of robot arm cause an inertia by the weight of joint actuator and rigid linkage when it changes in speed and/or direction. The modular design is an effective way to achieve a compact design. A mechanism can help reduce moving 
inertia by using five-bar mechanism for end-effector movement as shown in [4-6]. A cable transmission system can reduce energy consumption by relocating the actuators to the base [7]. Moreover, the cable can be used as a reduction mechanism with negligible backlash and low friction. A reduction of inertia makes change in motion easier, and reduce or prevent damage due to internal collisions. A cable transmission in robot arm is easier to move which robot has light weight structure [8-12]. In medical applications. Surgical robot system in MIS performs the 6-DOF motions that consists of the translational and rotational instrument for dexterous motion [13, 14]. Surgical robotic system which uses cable transmission performed in teleoperation [15].

A wire and pulley method are preferred components for transmitting a driving force [16-19]. The accuracy, size, and force are depending on joint mechanism so that a performance is improved by limited diameter. According to joint pulley, a tensioned cable winding at roller causes a moving relative joint which is following by capstan formula[20], Several previous works solved this problem by miniature joint mechanism such as in [21, 22]. However, a miniature part is difficult to fabrication and assembly. In limited workspace and choice of actuators, a remotely-actuated cable-driven mechanism is an alternative method in MRI compatibility for intraoperative surgical procedures. It has been used to perform needle insertion for prostate and thoracic intervention [23, 24]. In another type of cable transmission called tendon-sheath, bowden cable-driven robots are used in various applications such as neural rehabilitation [25, 26]. A cable is used to transmit force from proximal end to distal end and also control a position of cable which the length of the cable in the sheath is unchanged with friction $[27,28]$. The cable under tension reacts to straighten the cable conduit due to flexibility of conduit. However, an inefficiency and variations in cable tension occurs by friction and bending of conduit [29]. A specific control strategies become a solution to improve a tendonsheath in performance $[30,31]$.

Many sensors are required to improve an efficiency of transmission that causes a complex system. In wearable and rehabilitation, some researchers have attempted to build robotic rehabilitation devices based on cable-driven mechanisms [32-36]. Exoskeletons which attached to a human limbs provide movement function under rigid mechanical structure. In arm exoskeletons, a structure can be a serial/parallel rigid links with cable-suspended devices [37, 38]. By this method, joint misalignment between machine and human geometry can be avoided and reduced an injury to patient. The dynamics of the whole cable-driven system can be solved in multibody modeling and calculation of joint torques [39, 40]. A design of a multi-degree of freedom cable-driven exoskeleton was developed for the arm [41, 42]. According to a weight structure, a rigid links is replaced by lightweight cables in structure [43-45]. It has a low moving inertia on moving platform that is desirable in rehabilitation devices. However, a slackness and workspace of cable in control is still issue. The workspace analysis for cable-suspended mechanisms has been studied in [46-48]. This point need to be considered due to cable routing in structure. According to tension control, an end-effector force for functional rehabilitation is usually used a velocity control only $[49,50]$.

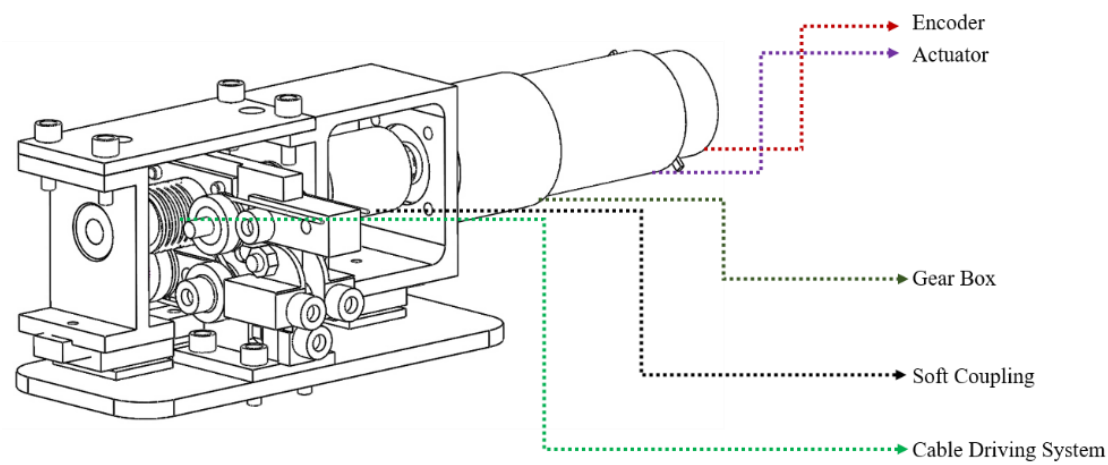

Figure 1. Cable driving System

In addition, various method of actuation including cable-driven is applied in soft wearable robots [51-54]. a consideration of soft tendon routing is pretension of the tendons without tendon derailment [55]. In addition, the improvement in accuracy measurement by using load cell which has been shown in [56-59]. In force feedback, haptic devices with cable is controlled by servo motors that generates a reaction force exert on users introduced in active robot $[60,61]$ and passive robot [62-64]. The lowest inertia is preferring to moving part so that a reaction force exert from device is more accuracy. In large footprint such as haptic device for stabilizing a double inverted pendulum use cable-driven to reduce a weight structure $[65,66]$. In 
specific area, the development of haptic devices is grasping control which is compatible with fMRI [67], surgical teleoperation $[68,69]$ and robotic hands $[70,71]$. In the other field, design a prototype of cabledriven multi stage was developed for mobile surveillance robot which can support various end-effector [72] while a design and consideration of precise cable drive for electro-optical tracking gimbals in [73, 74]. The effect of preload tension and backlash tested. Precise cable drive has been widely used in power transmission, which can provide a great improvement over gears, belts and pulleys, chains and sprockets and other alternative technologies. Significant advantages of precise cable drive include no lubrication, low friction, high stiffness, high efficiency and low cost [75].

In cable driving system, cable is reeled on the drum as driving part to transfers a force to a driven part. Therefore, it is possible in conversion of rotation-into-rotation (R2R) or rotation-into-translation (R2T). The distance of cable travelling is corresponding to the number of cable turn on the size of drum. The long stroke of transmission requires large size of drum and/or several number of cable turn. Although, the increasing number is possible but there is a limitation as well. Moreover, an increasing number of cable turn can cause an error and non-linear movement in operation. In precise motion of cable transmission, a complex calculation is needed. From these problems, the small size of drum can use in only short stroke in precision. Therefore, the compact cable transmission which has a high precision and long stroke is proposed in this paper as shown in Figure 1.

\section{MATERIALS AND METHODS}

\subsection{Principle of cable transmission}

In driving unit, the actuator shaft is connected to the drum which is a cylinder shape for winding several cable turns. The principle of cable transmission has an equivalent length of cable reel-in and reel-out in opposite direction similar to pull-push action. In static condition, the tension of both cable ends are the same whereas dynamic condition is difference. The applied torque from actuator generates an axial force on cable. The direction of rotation effects the tension of both cable ends. The different tension occurs by the friction between the cable and pulley and compliant of cable depending on direction of force as shown in Figure 2.
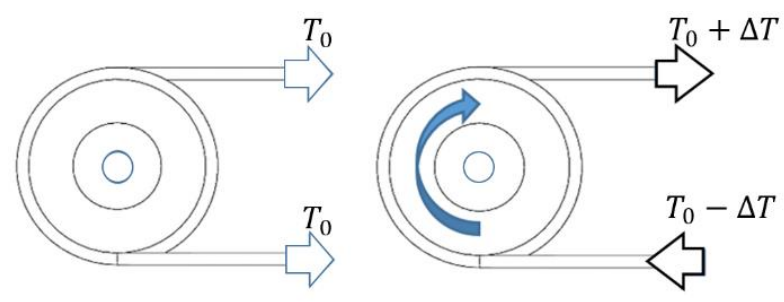

Figure 2. Working Principle of Cable Transmission

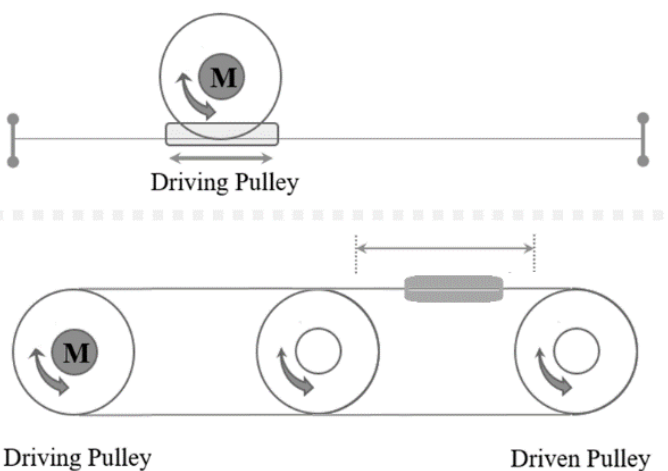

Figure 3. The type of driving motor: Moving base (top), Fixed base (bottom)

In mechanism, there are two possible ways to design a cable transmission for transferring the force in motion. The moving base type is quite constrained by the route of transmission similar to straight line because the cable which requires a high tension works as a rail for moving unit. On the other hand, the fixed base type is a driving unit is not moved but the cable is reeled in operation. It is appropriate with linear transmission, tendon-sheath transmission and in complex cable route which has the change of cable direction, see Figure 3. Moreover, it can be used in small moving part which can reduce a size of device at target or working area due to a small size of cable. In this paper, the fixed base type is considered for long stroke of operation and compact of driving system. In addition, the low inertia of cable moving causes a smooth motion along transmission and requires low watt of motor. In this case the lightweight of moving part can be designed by using this method. However, the specific design is required for complex structure

\subsection{Difficulty of cable transmission}


Cable transmission can be used in both linear and rotational motion in many approaches. The shape of pulley is depending on the purposes of usage such as roller, driving or driven pulley. Not only the type of pulley is concerned but the number of cable turn on the drum is a significant point for selection as well. Cable can be wound in less than one or more turns on the drum which has specific point of interest. Two major types of drum pulley in cable transmission are non-groove and groove as shown in Figure 4. Firstly, in non-groove type, the drum pulley has a smooth cylinder shape which allows a cable reels freely. The width of drum is corresponding to the cable size of travelling. It can be used in three ways; roller, driven or driving pulley. In roller, it is used in changing the direction of cable travelling which is usually winding less than one turn on drum. Moreover, the width of roller drum should fit to a cable diameter. In driven pulley, this function is quite similar to roller but the number of cable winding can have less than one or more depending on the applications such as in robot manipulator. Lastly, the driving pulley which is connected to the actuator shaft usually reel a cable in one or more turns to reel the cable for long stroke of movement. In addition, these three types show in Figure 5.

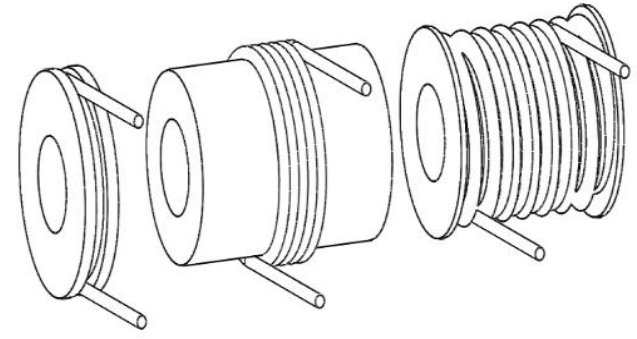

Figure 4. The type of pulley, non-groove and groove, half-turn (left) with groove, multi-turn with nongroove and multi-turn with groove (right)

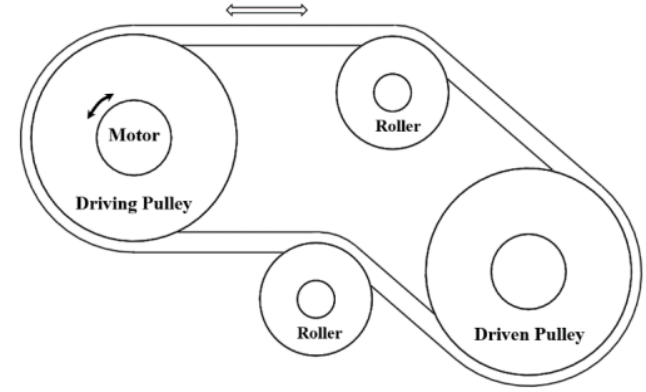

Figure 5. Three types of Pulley: driving, roller and driven pulley

Secondly, the groove type, the drum pulley has a helical or circular groove for constant transmission ratio. In this case, the drum pulley can work as a driving pulley or driven pulley. In this paper, the driving pulley is considered in a groove type. The reel-in and reel-out of cable is aligned in movement corresponding to number of turns. The travelling distance of cable transmission is relative to the circumference of the driving pulley. Thus, the large diameter can provide a long stroke of transmission.

The driving pulley which has a helical groove on the drum is reeled by cable. The rotation of actuator shaft reels-in and reel-out at the same distance in opposite direction. The increasing of number of turns generates the movement and effect of cable transmission. The number of turns which increases a fleet angle influences a non-linear movement in operation. The non-linear movement of cable is required a distance compensation as shown in Figure 6. Although, the complex compensation is possible to do but an increasing of number of turns increases a fleeting angle. Moreover, the length between two axes is also affect to the angle of fleet angle as shown in Figure 7. It is possible to have a small misalignment in small degrees which limits the number of turns. This angle causes an increasing friction on the surface of cable and adjacent cable on non-groove pulley and the surface of cable and the edge of the groove in groove pulley. In helical groove pulley, high value of angle is possible to cable jump to another groove.

The increasing of fleet angle causes the extension of length between two parallel axes. The nonlinear movement causes an error in several turns. In Figure. 6, a length $(l)$ has an addition length $\left(l_{e}\right)$ corresponding to the number of turn. In this case, the compensation is required for precise movement as follow:

$l_{n}=\sqrt{l_{0}^{2}+(n p)^{2}}$

The extended length is: $l_{e}=l_{n}-l_{0}$

The fleet angle is corresponding to the number of turns can be calculated as follow: $\alpha=\tan ^{-1} \frac{n p}{l}$

Where $\alpha=$ Fleet angle (degree)

$n=$ number of turns; $p=$ Pitch of helical groove; $l_{o}=$ length between driving axis and driven axis

$l_{n}=$ Length of incline angle, $\mathrm{n}=1,2,3 \ldots ; l_{e}=$ Extended Length 
In driving system, the size of driving pulley depends on the number of turn and diameter of driving pulley. The pulley requires large diameter to avoid a fleet long stroke movement. Normally, the fleet angle has an influence in cable transmission which it exists in increasing a rotation. The precision is corresponding to an incline angle of the cable at the driving pulley. The fleet angle is not increasing constantly by number of turns. The calculation and compensation are complex and difficult for making a precision of system and limitation of fleet angle. In addition, an increasing of pulley size which occupies a large volume is an alternative way to decrease the fleet angle for long stroke.

The fatigue and friction cause a reduction of life cycle of cable and cable transmission performance. In the pulley selection process, the size of cable (d), driving pulley (D) and cable construction are factors for design and consideration as shown in Fig. 8. This specification should be used in a design. In addition, the cable construction and dimeter ratio can be found from cable/drum manufacturer. The pulley diameter to cable diameter is recommended to be a ratio depending on the size of cable as which is a general rule for identification. This information guides to avoid greater fatigue or loose a flexibility of cable. However, there are other factors influence the system such as dynamic loading, differential loads in the cycle, drum alignment, groove profile, and fleet angle can have an effect on rope performance.

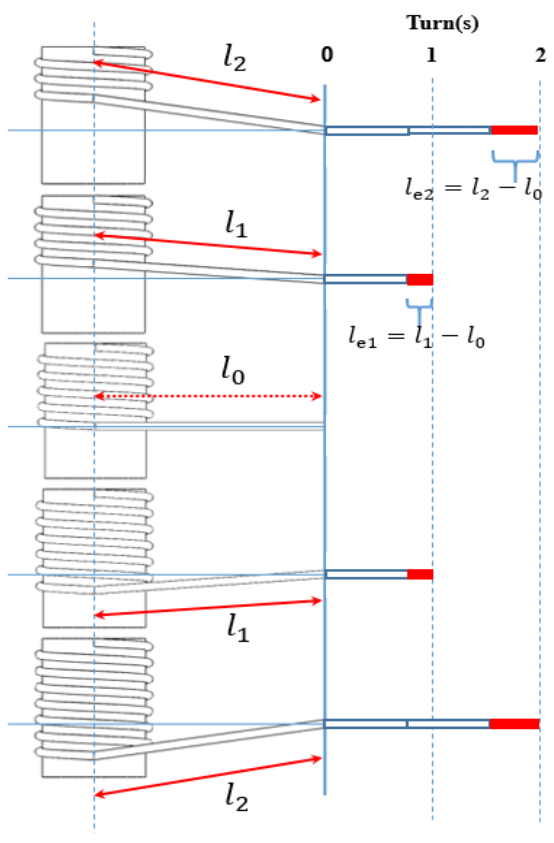

Figure 6. The multi-turn of cable transmission

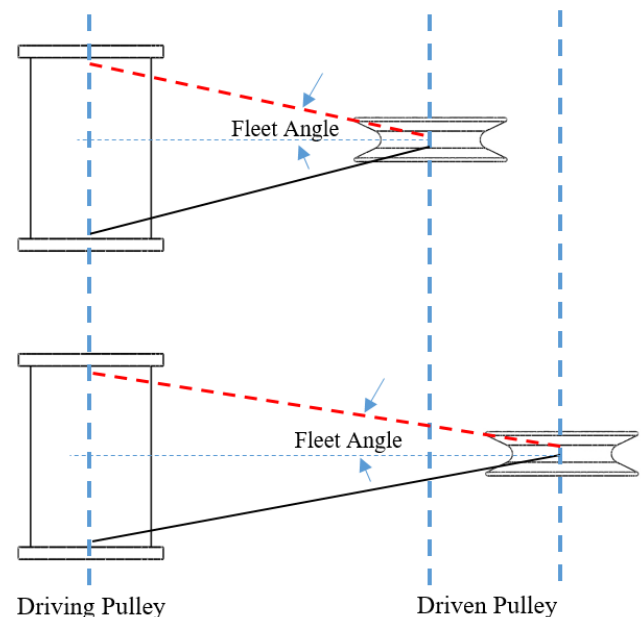

Figure 7. The Fleet Angle between two axes

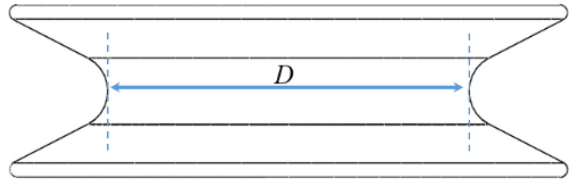

Pulley Diameter $(D)$

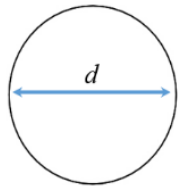

Cable Diameter $(d)$

Figure 8 . The schematic of cable and drum diameter

\section{DESIGN OF HIGH PRECISION OF CABLE TRANSMISSION SYSTEM}

In previous section, the constraint of cable transmission effects to the performance of system. The long stroke and compact size of cable transmission are purpose for the design. The drum pulley and cable size are the main key points for consideration. The fixed base type is selected for the driving unit. The groove 
type is a concept in design which pulley collects and releases a cable in the same distance to maintain the stability of system. In conventional cable drive, fleet angle exists in multiple turn of rotation. The new concept of design is introduced in this paper to eliminate the fleet angle which is a significant point of multiple turns of cable transmission as shown in Figure 9. According to this solution, the possibility of cable jump is solved as well. Consequently, cable transmission system will have a high performance in term of compactness and precision of driving system. The principle of cable winding on groove pulley has a same concept to the threaded rod or screw. A threaded rod, also known as a stud, is a long rod that is threaded on whole length or part of the rod. The thread can perform equivalent to the groove of pulley which is reeled by cable in several turns. However, a fleet angle still occurs if it is used similar way on the groove pulley. The relative motion of thread rod and number of cable turn are the key to solve this problem.

The specification of thread is depending on the size of the dimeter of rod and thread profile, see Table 1. In this process, the metric unit is convenient for calculation. The meaning the lead is the axial distance the screw moves in one revolution. The pitch is the axial distance between thread to thread. The most common standard is Unified and Matric thread. Most of them have right-handed thread which have coarse and fine types. Normally, the screw or bolt has a single-start thread which lead and pitch are the same axial distance. However, the multiple-start thread, the lead is equal to the pitch times the number of a start, see Figure 10. In this design, the size of selected cable should appropriate with the groove of thread profile. The layout of cable on the thread is shown in Figure 11.
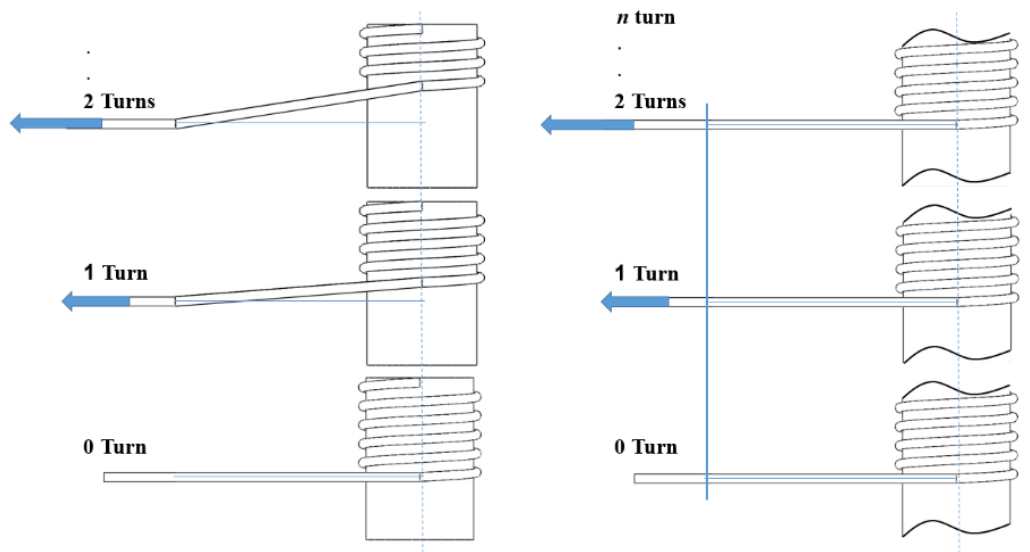

Figure 9. The desired output of cable feeding (right)

Table 1. Metric Thread Pitch and Tapping Sizes

\begin{tabular}{ccc}
\hline Size & PITCH, COARSE(MM) & Pitch, Fine $(\mathrm{mm})$ \\
\hline M10 & 1.5 & 1.25 \\
M12 & 1.75 & 1.25 \\
M16 & 2.0 & 1.5 \\
M20 & 2.5 & 1.5 \\
M24 & 3.0 & 2.0 \\
\hline
\end{tabular}
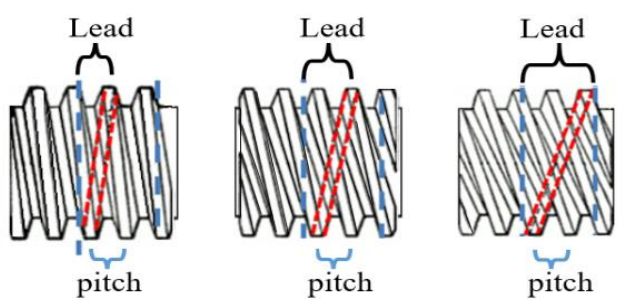

Figure 10.Relation of lead and pitch 


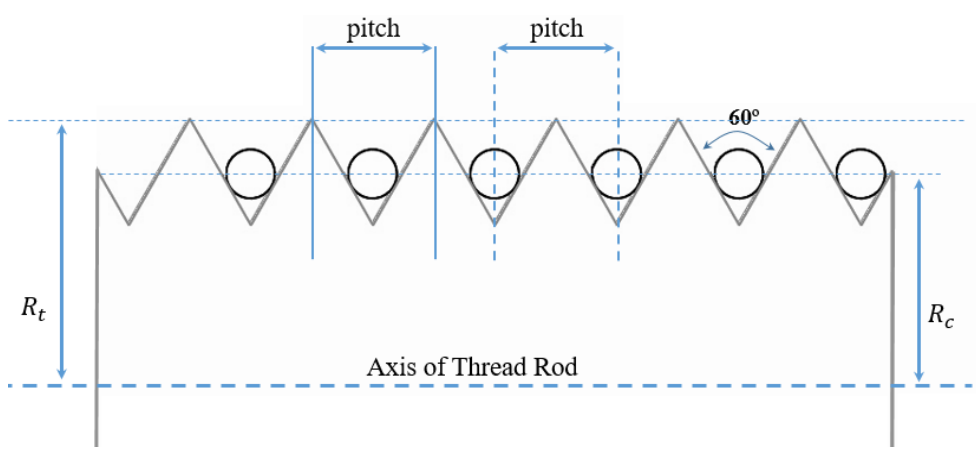

Figure 11. The thread profile and cable layout

In characteristic of cable transmission, reel-in and reel-out of cable on the drum is equivalent in rotational motion. The fleet angle is still existed in multiple turn of transmission which reduces a performance of cable transmission. In order to eliminate a fleet angle, the thread rod and the tap hole is used to avoid interference with itself in consecutive turn and reduced a cable-pulley friction. The module of both components should be the same in unit. In assembled parts, the revolution of the thread is converted to the translation of motion.

In Figure 12 shows the cable winding in the layout of left-to-right and right-to-left for the righthanded thread. In continuous motion, the reeled cable on the groove drum will change in both sides. For example, if the left side of cable winding is reeled-in for several turns which is the left-to-right form, the right side, right-to-left form, will be decreased in the same number of cable turn and vice versa. This method keeps an alignment of cable for continuous motion.
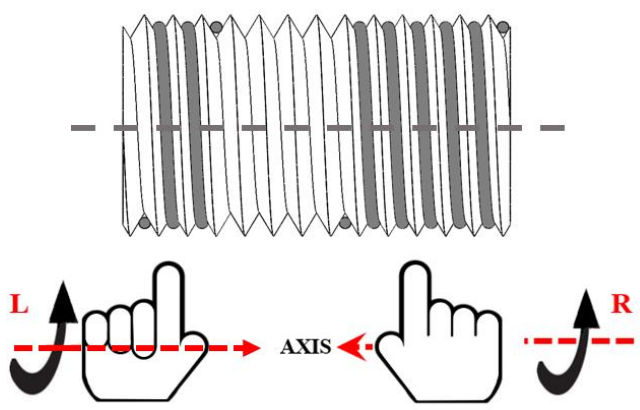

Figure 12. The form of rope on both sides, left to right and right to left (right-handed thread)

In long stroke of transmission, several turns are required for cable winding. The size of rod and stroke of cable can be calculated in following equation:

Helical Length of motor $\left(L_{H}\right) \mathrm{n} \operatorname{turn}(s) L_{H}=\sqrt[n]{p^{2}+C_{m}^{2}}$

Helical Length of angle movement of motor $L_{m}=\frac{\theta_{m}}{360} L_{H} L_{m}=\frac{\theta_{m}}{360} L_{H}$

The angle movement of driven pulley $L_{m}=\frac{\theta_{d}}{360} \pi d$

Relationship between $\theta_{d}$ and $\theta_{m} \theta_{d}=\frac{\theta_{m}}{\pi d} L_{H}$

Where

$C_{m}=$ Circumference of driving pulley; $p=$ Thread pitch; $\theta_{d}=$ Angle of driven pulley; $\theta_{m}=$ Angle of driving pulley; $d$ = diameter of driven pulley; $L_{m}=$ distance of angular movement

Figure 13 shows the prototype of CAD design of high precision of cable transmission system. The actuator connects to the thread rod as drum pulley in driving unit. One cable is used and the both end of cable is fixed at the drum pulley on both sides of edge. On distal end of thread rod have a bearing support for 
smooth rolling and stability. All of these parts are the set of moving part. The fixed part composes of the slide rail and the structure which has a tab hole. The tab hole should compatible with the thread rod is assembled in between both left-to-right and right-to-left. The number of turn is corresponding to the length of the thread rod and stroke requirement.

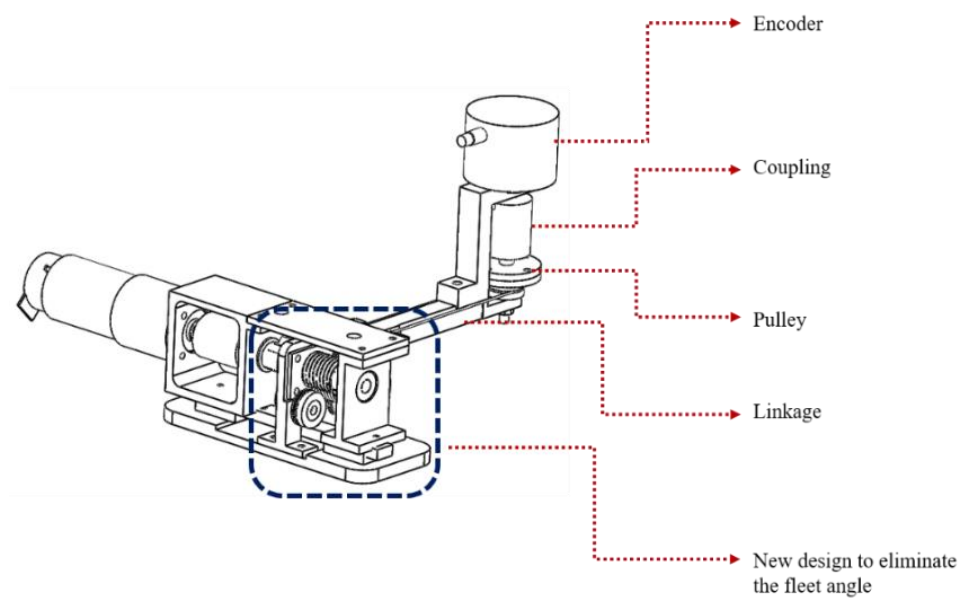

Figure 13. The thread profile and cable layout

\subsection{Tensioner}

One of significant factor for the performance of cable transmission is the tension of cable. Tensioner is the component which generates an axial force to cable. At rest, the tension should be adjusted in both side of cable ends. An increasing of tension generates higher friction or load in driving system. The appropriate adjustment should be used in an installation. Thus, the specific design and reliable is needed to use in the system. The adjustment is used by screwed bolt in design for self-locking purpose which does not affect to the system and convenient to setup. The alignment of the cable is the same after adjustment. In this design, a tensioner has on both sides on the base. In Figure 14 shows the structure and process of tensioner. In tension adjustment, if the bolt is adjusted by using hexagonal key, the roller pulley on the side of tensioner will be translated in horizontal plane. In this design, the cable will keep an alignment of cable after adjustment. After both side adjustment, both sides of tensioner have to be locked on the base of structure. In this mechanism, it is convenient to adjust a tension and ensure that it will not easily loose after adjustment.

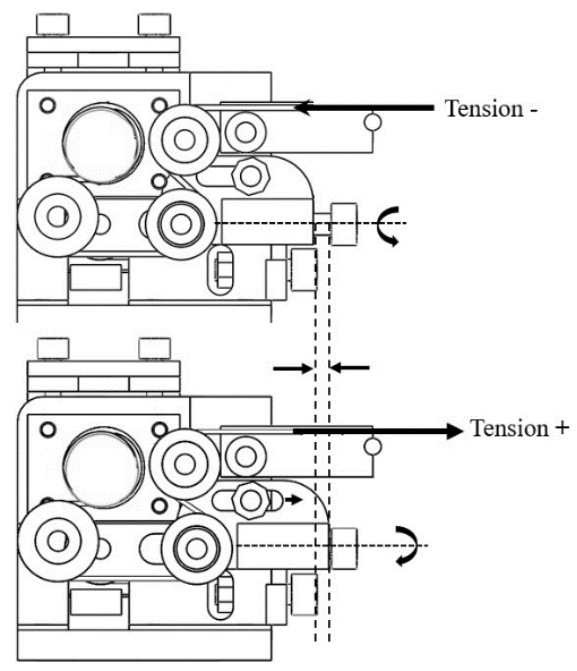

Figure 14. Tensioning process 


\section{EXPERIMENTS AND RESULTS}

In evaluation process, the high performance of cable transmission has to perform in term of accuracy and repeatability measurement of transmission system as shown in Figure 15 . The effect of the load in mechanism is also measured. The structure of system is composed of a sliding thread rod mechanism, actuator, controller and high resolution measuring component. The actuator which is MAXON DC motor with gearhead 246:1 reduction ratio is used. This actuator is connected to the MAXON EPOS2 which is a digital positioning controller.

The thread of the rod is M16 metric thread pitch $2.0 \mathrm{~mm}$, coarse pitch. This rod is modified both distal ends for locking cable end. The rod is connected to motor by coupling. The capacity of number of turns is maximum 15 turns which is possible to use only about 10 turns. The rests are for safety reason on both end of thread rod. The cable size is $0.8 \mathrm{~mm}$ of dimeter which is a $7 \times 7$ stainless steel wire rope sling. The tension cable can be measured by using tension gauge, Correx tension gauge and adjusted separately on both sides. In this system, the data will be collected at the joint of driven pulley which connects to the encoder. In addition, the rotary encoder has 1000 PPR resolution which has a 0.09 degree

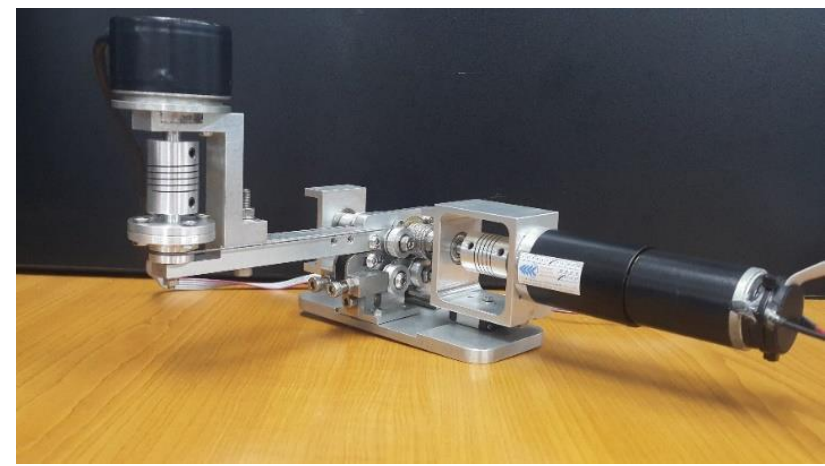

Figure 15. Command and Response in Forward Operation

\subsection{Load of system}

In all tests, the operation of motor starts from 0 turn and stop at 10 turns in forward direction. On the other hand, the reverse direction starts from 10 to 0 turns. In measurement, the trend of plot shows the load in operation in different speed of actuator and the overall friction along the range of thread. The first modality has only actuator measurement. In this case, actuator is measured a load or current to see the characteristic of actuator load in range of motion. Second modality, the actuator is connected with a thread rod which is assembled with a tab hole on the base. In this case, the friction is concerned due to an alignment of axis of rotation. The third and fourth modality, the all components includes a tensioned force T1 and T2 which have 100 and 250 centi newton, thus, the load in different tensions are displayed as shown in Figure 16 and 17. The average load in number of turn is measured to see the specific load of each operation as shown in Figure18. In the procedure, the speed of actuator is increasing from 500 to $2200 \mathrm{rpm}$ to see the load feedback in the range of operation. Four modalities are measured a current when the actuator is operated as shown in Table 2.

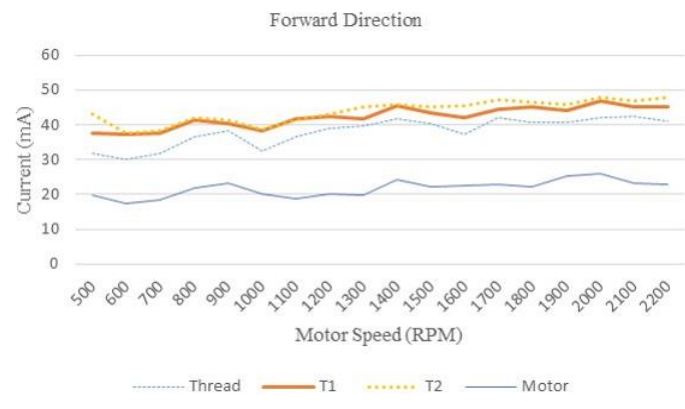

Figure 16. Command and Response in Forward Operation

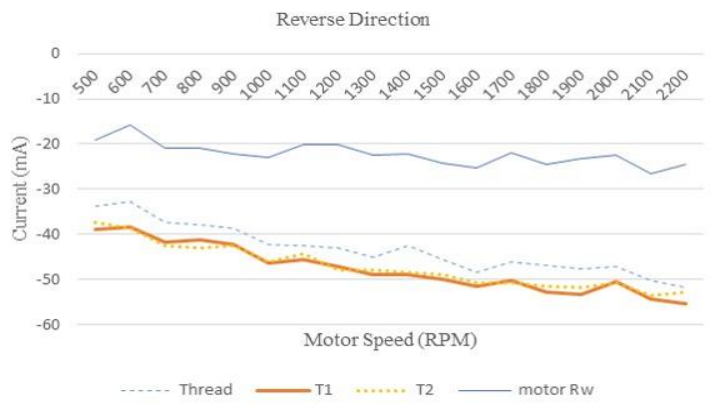

Figure 17. Command and Response in Reverse Operation 3 


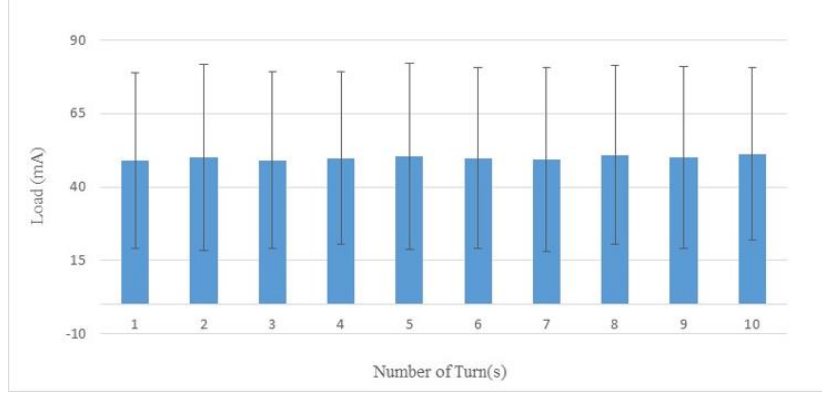

Figure 18. Average load in specific turn

Table 2. The modality of load measurement

\begin{tabular}{lllll}
\hline & Motor & Thread & Sling+T1 & Sling +T2 \\
\hline 1 & X & & & \\
2 & X & X & & \\
3 & X & X & X & X \\
4 & X & X & & \\
\hline
\end{tabular}

\subsection{Accuracy of System}

An accuracy of cable transmission is a significant point for performance of system. The compact system which has a long stroke of movement should guarantee for a precise movement. In measurement, the driven pulley is attached with rotary encoder to acquire a data of rotation. The high resolution of encoder provides a fine data in movement. The output result is measured by rotary encoder as an angle. The calculation of rotational angle of driven pulley can be converted to translation distance. Two aspects are considered to measure an accuracy of system. Firstly, the measurement in specific turn starts from zero turn to target turn which is a number 1 to 10 . One cycle is forward and backward direction of measurement. Secondly, the movement starts from zero turn and continue to next turn which one step increment has called continuous turn. The forward movement is finished until it reaches to a maximum turn. On the other hand, the backward movement starts from maximum turn to zero turn which has one step decrement. One cycle includes forward and backward as well. In both cases, all cycles are fixed at the same speed of rotation and tension of cable. These processes evaluate repeatability and accuracy of cable driving system. The result of specific and continuous turn of transmission system shows in Figure 19 and Figure 20, respectively. The repeatability and accuracy are performed to see and error in operation.

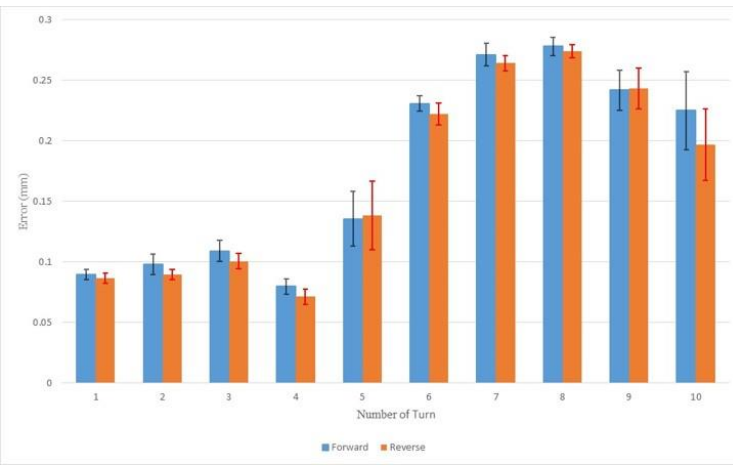

Figure 19. Error of Operation in specific turn

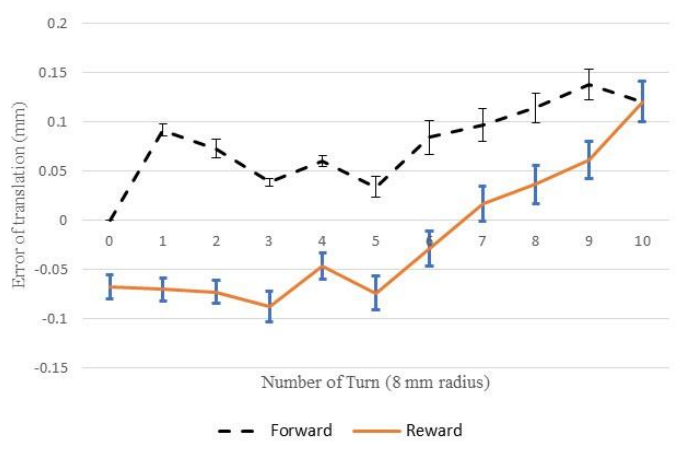

Figure 20. Error of Operation in Continuous turn

\section{DISCUSSION AND CONCLUSION}

A Cable transmission provides several advantages using wire and pulley system in aspect of compactness, lightweight and flexibility. It provides a high stiffness with small size, high strength, low friction, and low of backlash. These advantages are applied to many mechanical devices and applications. In transmission, pulley can be used in many aspects such as roller, driving and driven pulley. In each type, the purpose of selection is considered. The roller uses for changing direction of cable travelling. It is usually

Design of high performance DC motor actuated cable driving system ... (Chawaphol Direkwatana) 
wound less than one turn. However, in driving and driven purposes require one or several turns depending on the application. Conventional method is pull and push or cable length changes during movement of cable to generate a movement of rotation or translation. In this paper, the long translation is considered by using cable drive system which has driving pulley transmits to driven pulley. A groove pulley which cable is reeled on the drum maintains a pitch in several rotations. However, the fleet angle which is an incline angle along multiple cable turn is still occurs in multiple turn of cable driving pulley. Consequently, an increasing cable turn causes a nonlinear movement, cable fatigue and error desired position. The performance of cable transmission is not only relying on the tension of cable but the selection method of transmission is also influence on system. Therefore, an elimination of fleet angle is required in long stroke of cable transmission. In design, a groove pulley is used to maintain a continuous movement and guide a cable along a groove. However, the size of pulley and a fleet angle need to consider in driving system to avoid mentioned problems. A thread rod and tab hole which is a one degree of freedom by rotation to translation. In constant pitch of thread, a continuous motion is a relative motion on cable reel-in and reel-out. Consequently, in several cable turn, a fleet angle is eliminated on cable driving system. The high performance which provide a precise movement can be used by this method. In evaluation processes, a measurement of load and precision of system are considered.

In Load measurement, four modalities in forward, reverse and average load are measured to see each characteristic. In result of new design method shows a trend of load in difference speed of operation. An actuator which run without load has a slightly increased load in forward and reverse direction corresponding to a speed of rotation. On thread with tension has higher load of consumption. According to the trend of result, an increased external load consumes a power in similar way of speed of rotation which means a rotational friction along the thread rod is low and the load in specific turn has similar average. In thread with and without cable tension, the load from adding a tension is changed in small value which means an adding a tread rod method is not much effect to driving system.

In precision measurement, the position of several turns are considered. The forward and reverse motion are measured in specific moment and continuous movement to ensure a performance of cable driving system. A maximum turn is ten turns which is approximately to $50 \mathrm{~cm}$ of stroke of transmission. In a result of precision measurement, a low error of position control is proposed. In specific and continuous turn, an error is not constant along a transmission. The possibility of error causes by unsmooth surface, friction at stop position, Small Compliance of coupling at unsmooth point, error accumulation and misalignment of the structure. However, the new cable driving unit can perform in high precision of movement.

\section{ACKNOWLEDGEMENTS}

This research is financially support by National Research University Funds through Mahidol University and Government Research Budget through Mahidol University (Grant No. 111-2558), Thailand. Moreover, Sakol Nakdhamabhorn, Branesh Madhavan Pillai and BART LAB Researchers for their kind support.

\section{REFERENCES}

[1] Sung-Kyun Kim, Won-Ho Shin, Seong-Young KO, Jonathan Kim and Dong-Soo Kwo, "Design of a Compact 5DOF Surgical Robot of a Spherical Mechanism: CURES," International Conference on Advanced Intelligent Mechatronics, Xi'an, China, pp. 990-995, 2008.

[2] Won-Ho Shin and Dong-Soo Kwon, "Surgical Robot System for Single-Port Surgery with Novel Joint Mechanism," IEEE Transactions on Biomedical Engineering, Vol. 60, no. 4, pp. 937 - 944, 2013.

[3] Weihai Chen, Quanzhu Chen, Jianbin Zhang and Shouqian Yu, "Kinematics Control for a 7-DOF Cable-Driven Anthropomorphic Arm," International Conference on Intelligent Robots and Systems, Beijing, pp.1650-1655, 2006.

[4] H. Fang, L. Guo, and S. Bai, "A light weight arm designed with modular joints," Recent Advances in Mechanism Design for Robotics, vol. 33, pp. 47-54, 2015.

[5] L. Zhou, S. Bai, and M. R. Hansen, "Integrated dimensional and drivetrain design optimization of a lightweight anthropomorphic arm," Robotics and Autonomous Systems, vol. 60, no. 1, pp. 113-122, 2012.

[6] E. Cambell et al., "Design of a low-cost series elastic actuator for multirobot manipulation," IEEE International Conference on Robotic and Automation, Shanghai, China, pp. 5395-5400, 2011.

[7] Y.-J. Kim, J. Kim, J.-W. Lee, K.-M. Park, K.-S. Roh, and J.-Y. Choi, "RoboRay hand: A highly back drivable robotic hand with sensorless contact force measurements," IEEE International Conference on Robotic and Automation, pp. 6712-6718, 2014.

[8] T. Lens and O. von Stryk, "Investigation of safety in human-robot interaction for a series elastic, tendon-driven robot arm," IEEE/RSJ International Conference on Intelligent Robots and System, Vilamoura, Portugal, pp. 43094314, 2012. 
[9] M. Quigley, A. Asbeck, and A. Ng, “A low-cost compliant 7-DOF robotic manipulator," IEEE International Conference on Robotic and Automation, Shanghai, China, pp. 6051-6058, 2011.

[10] DongGyu Lee and TaeWon Seo, "Lightweight Multi-DOF Manipulator with Wire-Driven Gravity Compensation Mechanism," IEEE/ASME Transactions on Mechatronics, vol. 22, no. 3, pp. 1308 - 1314, 2017.

[11] Yong-Jae Kim, “Anthropomorphic Low-Inertia High-Stiffness Manipulator for High-Speed Safe Interaction,” IEEE Transactions on Robotics, vol. 33, no. 6, pp. 1358 - 1374, 2017.

[12] J. K. Salisbury and W. T. Townsend, "Compact cable transmission with cable differential," U.S. Patent 5207114A, May 5, 1993.

[13] C. Direkwatana, and J. Suthakorn, "Development of Wire-driven Laparoscopic Surgical Robotic System, MULapaRobot," IEEE International Conference on Robotics and Biomimetic, Thailand, pp 485-490, 2011.

[14] M. J. H. Lum, J. Rosen, M. N. Sinanan, and B. Hannaford, "Optimization of a spherical mechanism for a minimally invasive surgical robot: Theoretical and experimental approaches," IEEE Trans. Biomed. Eng., vol. 53, no. 7, pp. 1440-1445, 2006.

[15] S. A. Ali, M. F. Miskon, A. Z. H. Shukor, and, M. Q. Mhoammed, "The Effect of Parameters Variation on Bilateral Controller," International Journal of Power Electronics and Drive System (IJPEDS), vol. 9, no. 2, pp. 648-659, 2018.

[16] Mitchell J. H. Lum, Diana C. W. Friedman, Ganesh Sankaranarayanan, Hawkeye King, Kenneth Fodero II, Rainer Leuschke et al., "The RAVEN: Design and Validation of a Telesurgery System," The International Journal of Robotics Research, Vol. 28, No. 9, pp. 1183-1197, 2009.

[17] C. Ishii, K. Kobayashi, Y. Kamei, and Y. Nishitani, "Robotic forceps manipulator with a novel bending mechanism," IEEE/ASME Transection on Mechatronics, vol. 15, no. 5, pp. 671-684, 2010

[18] H. Yamashita, A. Iimura, E. Aoki, T. Suzuki, T. Nakazawa, E. Kobayashi, M. Hashizume, I. Sakuma, and T. Dohi, "Development of endoscopic forceps manipulator using multi-slider linkage mechanisms," Asian Symposium on Computer Aided Surgery-Robotic and Image Guided Surgery, Ibaraki, Japan, pp. 201-204, 2005.

[19] T. Y. Nai, J. L. Herder, and G. J. M. Tuijthof, "Steerable mechanical joint for high load transmission in minimally invasive instruments," ASME Journal of Medical Devices, vol. 5, pp. 034503-1-034503-6, 2011.

[20] L. E. Carlson, B. D. Veatch, and D. D. Frey, "Efficiency of prosthetic cable and housing," Journal of Prosthetics and Orthotics, vol. 7, no. 3, pp. 96-99, 1995.

[21] K. Nishizawa and K. Kishi, "Development of interference-free wire driven joint mechanism for surgical manipulator systems," Journal of Robotics and Mechatronics, vol. 16, no. 2, pp. 116-121, 2004.

[22] Changquing Li and Christopher D. Rahn, "Design of Continuous Backbone, Cable-Driven Robots," Journal of Mechanical Design, vol. 124, no. 2, pp. 265-271, 2002.

[23] Salih Abdelaziz, Laure Esteveny, Laurent Barbe, Pierre Renaud, Bernard Bayle, and Michel de Mathelin, "Development of a MRcompatible cable-driven manipulator," IEEE Conference on Robotics and Automation, Minnesota, USA, pp. 1488-1494, 2012.

[24] Nikolai Hungr, Ivan Bricault, Philippe Cinquin, and Celine Fouard, "Design and validation of a CT-and MRI-guided robot for percutaneous needle procedures," IEEE Transactions on Robotics, vol. 32, no. 4, pp. 973-987, 2016.

[25] Yeongjin Kim, Shing Shin Cheng, Aleksandrs Ecins, Cornelia Fermuller, Kelly P Westlake, and Jaydev P Desai, "Towards a robotic hand rehabilitation exoskeleton for stroke therapy," ASME 2014 Dynamic Systems and Control Conference, Texas, USA, pp. V001T04A006; 9 pages, 2014.

[26] Tian Fuxiang and Wang Xingsong, "The design of a tendon-sheath driven robot," International journal of intelligent systems technologies and applications, vol. 8, no.1, pp.215-230, 2009.

[27] Chen Lin, Wang Xingsong, and Tian Fuxiang, "Tendon-sheath actuated robots and transmission system," International Conference on Mechatronics and Automation, Changchun, China, pp. 3173-3178, 2009.

[28] Soo J Phee, SC Low, Paolo Dario, and Arianna Menciassi, "Tendon sheath analysis for estimation of distal end force and elongation for sensorless distal end," Robotica, vol, 28, no. 07, pp.1073-1082, 2010.

[29] M. H. Letier, P. A. Schiele, M. Avraam, M. Horodinca, and A. Preumont, "Bowden cable actuator for torquefeedback in haptic applications," Proc. Eurohaptics, pp. 1-6, 2006.

[30] K. Kong, J. Bae, and M. Tomizuka, "Torque mode control of a cabledriven actuating system by sensor fusion," Journal of Dynamic System, Measurement, and control, vol. 135, no. 3, pp.031003, 2013.

[31] V.Agrawal, W.J. Peine and B. Yao, "Modeling of transmission characteristics across a cable-conduit system," IEEE Transaction on Robotics, vol. 26, no. 5, pp. 914-924, 2010.

[32] Y. Mao and S. K. Agrawal, "A cable driven upper arm exoskeleton for upper extremity rehabilitation," IEEE Conference on Robot and Automation, Shanghai, China, pp. 4163-4168, 2011.

[33] Ying Mao and Sunil Kumar Agrawal, "Design of a Cable-Driven Arm Exoskeleton (CAREX) for Neural Rehabilitation," IEEE Transactions on Robotics, vol. 28, no. 4, pp. 922 - 931, 2012.

[34] G. Rosati, M. Andreolli, A. Biondi, and P. Gallin, "Performance of cable suspended robots for upper limb rehabilitation," IEEE International conference on Rehabilitation Robotics, Netherlands, pp. 385-392, 2007.

[35] K. Homma, O. Fukuda, J. Sugawara, Y. Nagata, and M. Usuba, "A wiredriven leg rehabilitation system: Development of a 4-DOF experimental system," IEEE/ASME International Conference on Advanced Intelligent Mechatronics, Japan, pp. 908-913, 2003.

[36] M. Otis, M. Mokhtari, C. du Tremblay, D. Laurendeau, F. De Rainville, and C. Gosselin, "Hybrid control with multi-contact interactions for 6DOF haptic foot platform on a cable-driven locomotion interface," Symposium on Haptic Interfaces for Virtual Environment and Teleoperator Systems, USA, pp. 161-168, 2008. 
[37] Xiang Cui, Weihai Chen, Xin Jin, Sunil K. Agrawal, "Design of a 7-DOF Cable-Driven Arm Exoskeleton (CAREX7) and a Controller for Dexterous Motion Training or Assistance," IEEE/ASME Transactions on Mechatronics, vol. 22, no. 1, pp. 161-172, 2017.

[38] G. Rosati, P. Gallina, and S. Masiero, "Design, implementation and clinical tests of a wire-based robot for neurorehabilitation," IEEE Trans. Neural Syst. Rehabil. Eng., vol. 15, no. 4, pp. 560-569, 2007.

[39] S. Mustafa and S. K. Agrawal, "On the force-closure analysis of n-DOF cable-driven open chains based on reciprocal screw theory," IEEE Transaction on Robotics, vol. 28, no. 1, pp. 22-31, 2012

[40] J. Wang, X. Cui, W. Chen, and Y. Jin, "Dynamic analysis of cable-driven humanoid arm based on Lagrange's equation," Advances in Reconfigurable Mechanisms and Robots I, J. S. Dai, M. Zoppi, and X. Kong, Eds. London, U.K.: Springer, 2012, pp. 619-628.

[41] G. Yang, H. L. Ho, W. Chen, W. Lin, S. H. Yeo, and M. Kurbanhusen, "A haptic device wearable on a human arm," IEEE conference on Robotics, Automation and Mechatronics, Singapore, pp. 243-247, 2004.

[42] E. Brackbill, Y. Mao, S. Agrawal, M. Annapragada, and V. Dubey, "Dynamics and control of a 4-DOF wearable cable-driven upper arm exoskeleton," IEEE International Conference on Robotics and Automation, Kobe, Japan, pp. 2300-2305, 2009.

[43] S. Oh and S. Agrawal, "Cable suspended planar robots with redundant cables: Controllers with positive tensions," IEEE Transaction on Robotics, vol. 21, no. 3, pp. 457-465, 2005.

[44] S. Oh and S. Agrawal, "Generation of feasible set points and control of a cable robot," IEEE Transaction on Robotics, vol. 22, no. 3, pp. 551-558, 2006.

[45] S. Oh and S. Agrawal, "The feasible workspace analysis of a set point control for a cable-suspended robot with input constraints and disturbances," IEEE Transaction on Control System Technology, vol. 14, no. 4, pp. 735-742, 2006.

[46] M. Gouttefarde and C. M. Gosselin, "Analysis of the wrench-closure workspace of planar parallel cable-driven mechanisms," IEEE Transaction on Robotics, vol. 22, no. 3, pp. 434-445, 2006.

[47] C. B. Pham, S. H. Yeo, G. Yang, M. S. Kurbanhusen, and I. M. Chen, "Force-closure workspace analysis of cabledriven parallel mechanisms," Mechanism and Machine Theory, vol. 41, pp. 53-69, 2006.

[48] S. Agrawal, X. Chen, J. Galloway, "Training Special Needs Infants to Drive Mobile Robots Using Force-Feedback Joystick," IEEE International Conference on Robotics and Automation, pp. 4797-4802, 2010.

[49] F. J. Castillo-Garcia, P. Rea, A. Gonzalez-Rodriguez, E. Ottaviano, "On the Design of a 4 Degrees-of-freedom Pick and Place Cable Suspended Parallel Manipulator," International Journal of Robotics and Automation, Vol.6, pp.286-302, 2017.

[50] K. A. Witte, J. Zhang, R. W. Jackson, and S. H. Collins, "Design of two lightweight, high-bandwidth torquecontrolled ankle exoskeletons," IEEE International Conference on Robotics and Automation, USA, pp. 1223-1228, 2015.

[51] Hyunki In, Useok Jeong, Haemin Lee, Kyu-Jin Cho, "A Novel Slack-Enabling Tendon Drive That Improves Efficiency, Size, and Safety in Soft Wearable Robots," IEEE/ASME Transactions on Mechatronics, vol. 22, no. 1, pp. 56-70, 2017.

[52] S. Lee, K. A. Landers, and H.-S. Park, "Development of a biomimetic hand exotendon device (BiomHED) for restoration of functional hand movement post-stroke," IEEE Trans. Neural Syst. Rehabil. Eng., vol. 22, no. 4, pp. 886-898, 2014.

[53] Y.-L. Park et al., "Design and control of a bio-inspired soft wearable robotic device for ankle-foot rehabilitation," Bioinspir. Biomim. vol. 9, no. 1, Art. 016007, 2014.

[54] V. Bartenbach, K. Schmidt, M. Naef, D. Wyss, and R. Riener, "Concept of a soft exosuit for the support of leg function in rehabilitation," IEEE International Conference on Rehabilitation Robotics, Singapore, pp. 125-130, 2015.

[55] H. In, B. B. Kang, M. Sin, and K. Cho, "Exo-Glove: Soft wearable robot for the hand using soft tendon routing system," IEEE Robotics and Automation magazine, vol. 22, no. 1, pp. 97-105, 2015.

[56] S. Ball, I. Brown, and S. Scott, "Medarm: a rehabilitation robot with 5dof at the shoulder complex," IEEE/ASME international conference on Advanced intelligent mechatronics, pp. 1-6, 2007.

[57] S. K. Banala, S. H. Kim, S. K. Agrawal, and J. P. Scholz, "Robot assisted gait training with active leg exoskeleton (alex)," IEEE Trans. Neural Syst. Rehabil. Eng., vol. 17, no. 1, pp. 2-8, 2008.

[58] J. Emken, R. Benitez, and D. Reinkensmeyer, "Human-robot cooperative movement training: Learning a novel sensory motor transformation during walking with robotic assistance-as-needed," J. Neuroeng. Rehabil. vol. 4, no. 8, pp. 1-16, 2007.

[59] Duschau-Wicke, J. von Zitzewitz, A. Caprez, L. Lunenburger, and R. Riene, "Path control: A method for patientcooperative robot-aided gait rehabilitation," IEEE Trans. Neural Syst. Rehabil. Eng., vol. 18, no. 1, pp. 38-48, 2010.

[60] S. Kawamura and K. Ito, "A New Type of Master Robot for Teleoperation Using A Radial Wire Drive System," IEEE/RSJ International Conference on Intelligent Robots and Systems, vol. 1, pp. 55-60, 1993.

[61] J. V. Zitzewitz, G. Rauter, R. Steiner, A. Brunschweiler, and R. Riener, "A Versatile Wire Robot Concept as a Haptic Interface for Sport Simulation," IEEE International Conference on Robotics and Automation, pp.313-318, 2009.

[62] T. Koyama, I. Yamano, K. Takemura, and T. Maeno, "Multi-Fingered Exoskeleton Haptic Device using Passive Force Feedback for Dexterous Teleoperation," IEEE/RSJ International Conference on Intelligent Robots and Systems, pp. 2905-2910, 2002. 
[63] B. M. Pillai, and J. Suthakorn, "Motion Control Applications: Observer Based DC Motor Parameters Estimation for Novices," International Journal of Power Electronics and Drive Systems (IJPEDS), vol. 10, no. 1, pp. 195-210, 2019.

[64] Y. Hirata, K. Suzuki, and K. Kosuge, "Improvement in the Performance of Passive Motion Support System," IEEE International Conference on Robotics and Automation, pp. 4272-4277, 2011.

[65] Joan Savall, Javier Martín and Alejo Avello, "High-performance linear cable transmission," Journal of Mechanical Design, vol. 130, no.6, pp. 064501 (5 pages), 2008.

[66] Jiun-Yih Kuan, Kenneth A. Pasch, and Hugh M. Herr, "A High-Performance Cable-Drive Module for the Development of Wearable Devices," IEEE/ASME Transactions on Mechatronics, vol. 23, no. 3, pp. 1238 - 1248, 2018.

[67] Bogdan Vigar, James Sulzer, Roger Gassert, "Design and Evaluation of a Cable-Driven fMRI-Compatible Haptic Interface to Investigate Precision Grip Control," IEEE transactions on haptics, vol. 9, no. 1, pp.20-32, 2016.

[68] C. H. King, M. O. Culjat, M. L. Franco, C. E. Lewis, E. P. Dutson, W. S. Grundfest, and J. W. Bisley, "Tactile feedback induces reduced grasping force in robot-assisted surgery," IEEE Trans. Haptics, vol. 2, no. 2, pp. 103-110, 2009.

[69] B.M. Pillai, C. Wilasrusmee and J. Suthakorn, "Observer Based Dynamic Control Model for Bilaterally Controlled MU-LapaRobot: Surgical Tool Force Limiting," International Journal of Electrical and Computer Engineering, vol. 10, no.1, pp. 828-839, 2020.

[70] Changsheng Li, Xiaoyi GU and Hongliang Ren, "A Cable-Driven Flexible Robotic Grasper with Lego-Like Modular and Reconfigurable Joints," IEEE/ASME Transactions on Mechatronics, vol. 22, no. 6, pp. 2757 - 2767 , 2017.

[71] Tomohiro Nakano, Yuki Saito and Takahiro Nozaki, and Kouhei Ohnishi, "Variable Tension Control for MasterSlave Tendon-Driven Robot Hand," International Conference on Mechatronics (ICM), pp. 588-593, 2013.

[72] Jianjun Yuan, Weiwei Wan, Kaiwei Chen, Qi Fang and Weijun Zhang, "Design and Prototyping a Cable-driven Multi-stage Telescopic Arm for Mobile Surveillance Robots," IEEE International Conference on Robotics and Biomimetics, pp.1845-1850, 2014.

[73] D. Lee, D. Chang, Y. IL Shin, D. Son, T. wan Kim, K.-Y. Lee, and J. Kim, "Design and application of a wire-driven bidirectional telescopic mechanism for workspace expansion with a focus on shipbuilding tasks," Advanced Robotics, vol. 25, pp. 699-175, 2011.

[74] Yafei Lu, Dapeng Fan, "Transmission backlash of precise cable drive system," Journal of Mechanical Engineering Science, vol. 227, no. 10, pp. 2256-2267, 2012.

[75] C. Bascom, J.R. Stewart and L.Y. Tang, "Evaluation of pulling rope wear and coefficient of friction for pipe-type cables," IEEE Transactions on Power Delivery, vol. 12, no 2, pp. 542-546, 1997. 